\title{
Neurovascular compression of the trigeminal and glossopharyngeal nerve: three case reports
}

\author{
A-M Childs, J F Meaney, C D Ferrie, P C Holland
}

\begin{abstract}
Trigeminal neuralgia (TN) is a frequent cause of paroxysmal facial pain and headache in adults. Glossopharyngeal neuralgia (GPN) is less common, but can cause severe episodic pain in the ear and throat. Neurovascular compression of the appropriate cranial nerve as it leaves the brain stem is responsible for the symptoms in many patients, and neurosurgical decompression of the nerve is now a well accepted treatment in adults with both TN and GPN who fail to respond to drug therapy. Neither TN nor GPN are routinely considered in the differential diagnosis when assessing children with paroxysmal facial or head pain, as they are not reported to occur in childhood. Case reports of three children with documented neurovascular compression causing severe neuralgic pain and disability are presented. The fact that these conditions do occur in the paediatric population, albeit rarely, is highlighted, and appropriate investigation and management are discussed.

(Arch Dis Child 2000;82:311-315)
\end{abstract}

Keywords: trigeminal neuralgia; glossopharyngeal neuralgia; facial pain; headache; microvascular decompression; Gabapentin

Trigeminal neuralgia (TN) is a relatively common cause of facial pain in adults, typically presenting in the fourth or fifth decade. The pain is paroxysmal, usually unilateral, and affects one or more divisions of the fifth cranial nerve. The most commonly affected single division is the mandibular branch, with the ophthalmic division the least often affected. The pain is often triggered by minimal stimulation of the affected area. Although pain may be experienced frequently throughout the day it characteristically does not occur at night. ${ }^{12}$ Glossopharyngeal neuralgia (GPN), characterised by bursts of severe, unilateral, lancinating pain of the same quality as that in $\mathrm{TN}$, occurs much less frequently than $\mathrm{TN}$ in the adult population, with a peak incidence between 40 and 60 years. ${ }^{12}$ Two forms seem to exist: one where the pain is mainly in the tonsillar area and throat, and the other where pain is felt deep in the ear. Mechanical stimulation of the fauces by swallowing, talking, and coughing typically trigger the pain. ${ }^{1-3}$

TN can be caused by tumours, ${ }^{4}$ vascular malformation, ${ }^{45}$ or multiple sclerosis. ${ }^{67}$ However, most cases are idiopathic and not associated with abnormalities on conventional mag- netic resonance (MR) imaging. There is now convincing evidence that in these so called idiopathic cases pain results from vascular compression of the nerve root, typically occurring in older patients in whom atherosclerotic elongation brings arteries in contact with the Vth nerve as it leaves the pons. Veins are also, though less commonly, implicated. ${ }^{8} 9$ Although GPN is a much rarer condition, based on numerous anecdotal reports it is believed that in the absence of a tumour, the idiopathic form may also result from arterial compression of the nerve as it leaves the brain stem. ${ }^{10-12}$

Much of the scepticism regarding the aetiological role of neurovascular compression (NVC) in TN has abated for two reasons. Firstly, numerous large surgical series consistently describe the occurrence of NVC and the success of microvascular decompression (MVD) in TN. ${ }^{13-15}$ Secondly, by exploiting advances in high resolution MR angiography, which allows demonstration of the exact relation of the pons and trigeminal nerves to the adjacent vessels, several authors have reported a high incidence of NVC in TN but a very low incidence in asymptomatic subjects. ${ }^{16-18}$ Therefore the identification of patients suitable for neurosurgical treatment with MVD is now possible.

Although the causal association between neurovascular compression and $\mathrm{TN}$ is now less contentious, the age at which patients may be affected is more controversial. Adult neurological texts refer to idiopathic TN occurring occasionally in childhood, ${ }^{1}$ and a number of children have undergone successful MVD in the USA. ${ }^{19}$ However, there are few reports of TN occurring in paediatric literature; indeed there is debate as to whether or not it presents in children at all and there are no reports of GPN affecting children.

Here we describe the case histories of three children who presented over a two year period with severe, debilitating facial pain as a result of neurovascular compression of their cranial nerves.

\section{Case 1}

A 9 year old boy, previously fit and well, had a 10 month history of right sided headache. The pain was precipitated by movement and triggered by any stimulation of the right side of his forehead. He was unable to wash or comb his hair and had difficulty sitting up or walking. The pain occurred in sudden bursts at which time he would grimace and writhe on the bed. The pain had become increasingly severe, with 30-60 second lightning bursts every two minutes. At this stage he was essentially 
bedridden and had difficulty communicating. However, the pain always disappeared at night.

On examination he had notable hyperaesthesia from the right supraorbital margin into his hairline, but not crossing the midline or extending posteriorly. There were no other positive examination findings. In particular, he was normotensive, had normal fundoscopic appearances, and intact corneal reflexes. Dental examination and psychological evaluation did not reveal any abnormalities.

Viral titres, including herpes, were negative on three occasions. Full blood count, viscosity, and $\mathrm{C}$ reactive protein were all within normal limits. Autoantibodies were not detected. A lumbar puncture revealed no cells, normal CSF protein, and no oligoclonal bands. Computed tomography (CT) with contrast and conventional spin echo MR imaging did not show any intracranial abnormality. However, MR angiography was not performed at this stage.

His symptoms did not respond to simple oral analgesics or intravenous morphine. High dose carbamazepine had a transient beneficial effect. Prolonged, high dose courses of amitriptyline, phenytoin, baclofen, sodium valproate, sumatriptan, and acyclovir, together with a three day course of methylprednisolone were all ineffective. Three separate stellate ganglion injections had no effect on his pain.

\section{PROGRESS}

Although the history and examination findings were most suggestive of unilateral $\mathrm{TN}$ affecting the ophthalmic division of the nerve, alternative diagnoses and treatment strategies were pursued because of the reluctance to accept the diagnosis of TN at his age. Because of failure of all other therapies, exploratory posterior fossa craniotomy was performed, 14 months after his initial presentation. This revealed arterial compression of the Vth nerve exiting the pons with surrounding adhesions and some venous congestion. Postoperatively, the frequency of his attacks reduced. However, within two months the pain had returned to its previous intensity and he remained bedridden.

$\mathrm{He}$ was referred to an internationally renowned centre in Pittsburgh in the USA, where he underwent two further craniotomies within a week. On the first occasion two petrosal veins were removed from the surface of the nerve but no recovery was evident. On the second occasion a venule, missed during the earlier operation, was identified entering the posterior aspect of the nerve and was ligated.

Over subsequent weeks the episodes of pain subsided. The child was able to return to school and was almost pain free on carbamezapine and baclofen. He did experience painful spasms in association with intercurrent infections and these became more severe over the next six months. These pains were identical to those experienced previously and examination findings of hyperaesthesia in the ophthalmic division of the trigeminal nerve remained constant. MR angiography showed no evidence of compression or distortion of the nerve.

A fourth craniotomy was performed, once again in Pittsburgh, and an abnormal artery and vein were found to be compressing the nerve, which was decompressed. Thirteen months after this operation the child is completely pain free, off all medication, and has resumed his normal activities.

\section{Case 2}

A 12 year old boy presented with an eight week history of severe lightning pain affecting his left forehead, cheek, and preauricular area occasionally radiating to the mandible. The pain never extended across the midline or into the hairline. The pain came in 1-5 minute waves, during which he would cry out, become pale, and lie down. Attacks occurred up to 15 times a day, in increasingly severe clusters when they were followed by a bilateral frontal headache. $\mathrm{He}$ was unable to tolerate being touched on the left forehead or cheek. He could not clean his teeth or brush his hair and was finding it difficult to eat. He never experienced pain during sleep.

On examination he had a well defined area of hyperaesthesia over the left forehead and cheek which did not cross the midline. $\mathrm{He}$ was normotensive. There were no other abnormal examination findings.

Routine blood tests were negative with no evidence of inflammation. His CSF cell count and protein were normal. CT scans and conventional spin echo MR imaging of brain and sinuses were normal. MR angiography revealed grooving of the lateral aspect of the left trigeminal nerve by a sizeable vein (fig 1 ).
A

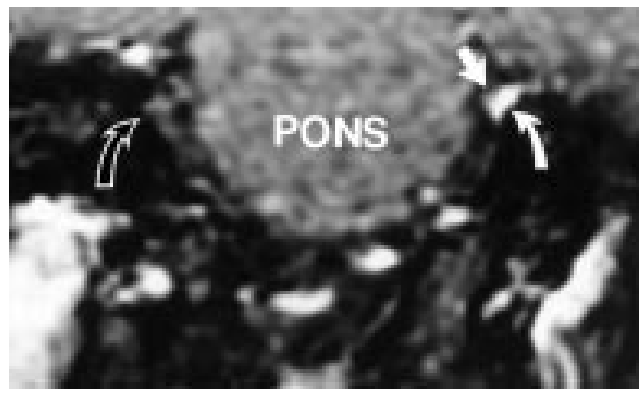

B

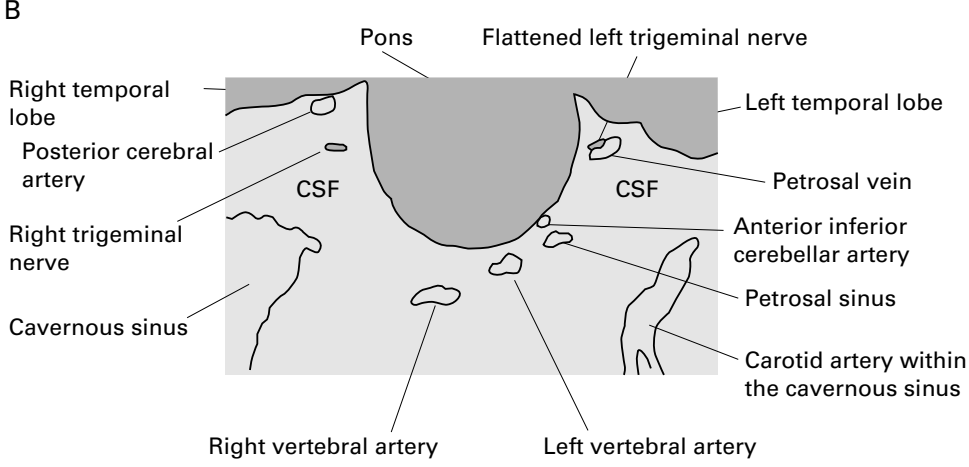

Figure 1 (A) Coronal format from contrast enhanced 3D MR angiogram shows compression and flattening of the left trigeminal nerve (straight arrow) by the petrosal vein (solid, curved arrow). No evidence of compression of the right trigeminal nerve (open, curved arrow). (B) Line diagram showing MR features. 
A

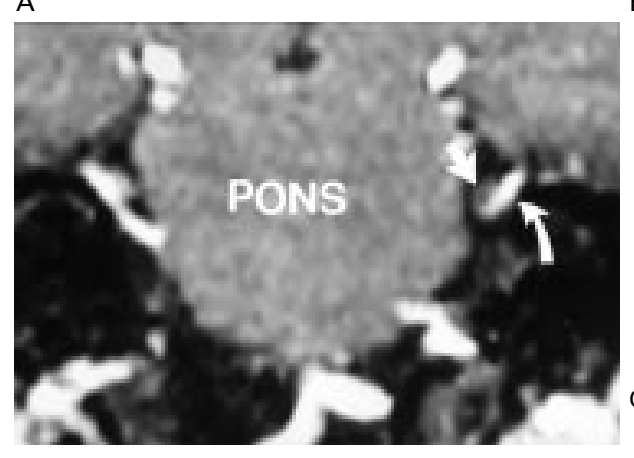

B

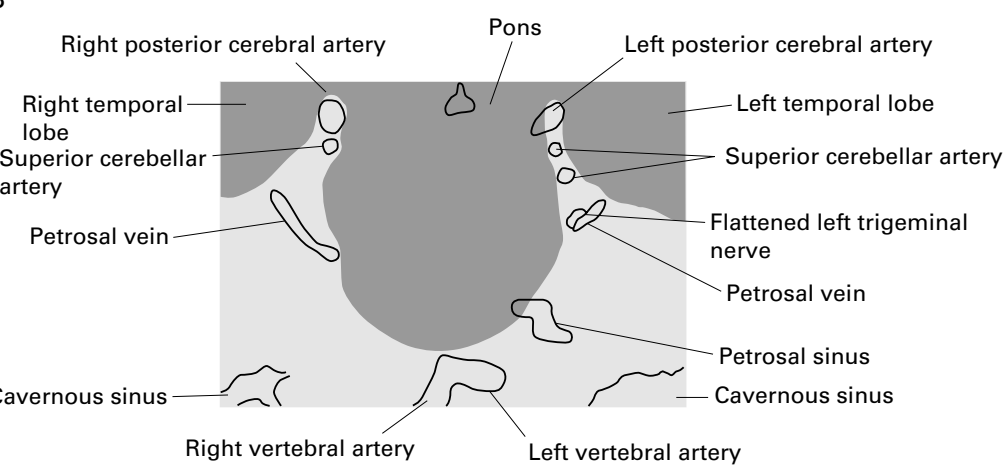

Figure 2 (A) Coronal reformat five months later, following attempted decompression, shows persisting compression of the lateral aspect of the nerve, essentially unchanged from preoperative scan (fig 1). On the right side, the petrosal vein runs in close proximity to the trigeminal nerve. Inspection of the right nerve along its length reveals no evidence of compression. Curved arrow, petrosal vein; straight arrow, left trigeminal nerve. (B) Line diagram showing MR features.

PROGRESS

Conventional analgesia in high dosage had been used prior to the diagnosis of TN with no effect on his symptoms. Following diagnosis, high dose carbamezapine and baclofen were tried but with minimal benefit.

Given this lack of response to conventional treatment and the positive findings at MR angiography, he was referred for microvascular decompression. At operation, a vein was found crossing the lateral aspect of the nerve root proximally and was removed. There was some relief of the symptoms postoperatively. However within 48 hours the pain recurred again.

Five months postoperatively the lightning pains were occurring up to 25 times a day. $\mathrm{He}$ was reluctant to eat, drink, or mobilise and had not attended school for six months. He continued to experience generalised frontal headache after severe bursts of unilateral pain. Repeat MR angiography showed persistent deformation of the left trigeminal nerve by the vessel identified previously (fig 2). Further surgery to decompress the nerve was undertaken.

He had a difficult postoperative course with severe dizziness and headache. However, these symptoms resolved and his severe bursts of pains first diminished and are now absent. $\mathrm{He}$ is no longer on medication and attends full time school. Repeat MR angiography showed that the previous abnormal vessel was no longer present.

\section{Case 3}

A 13 year old girl presented with a history of paroxysmal pain in the right ear from infancy. For years this was attributed to otitis media, often with no clinical evidence of inflammation. Neither antibiotics nor conventional analgesia provided any symptom relief. The pain was characteristically of sudden onset, with no obvious precipitant and very severe. Attacks lasted from a few seconds to over an hour and occurred three to four times a month. During attacks she would cry out in distress and need to lie down. She occasionally experienced dizziness and tinnitus but not in association with the pain in her ear. Her hearing was normal. She was normotensive and both systemic and neurological examination were normal.

MR of the brain was normal, but MR angiography revealed a prominent, looping right posterior inferior cerebellar artery, compressing the right IX/Xth nerve complex at its exit from the medulla (figs 3 and 4).

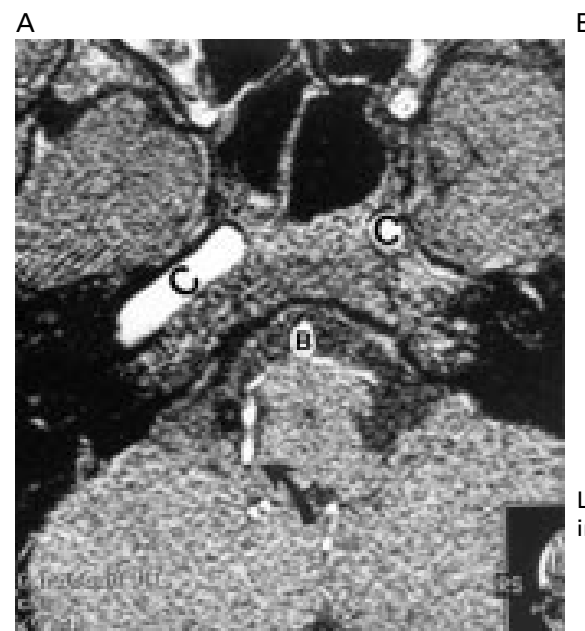

B

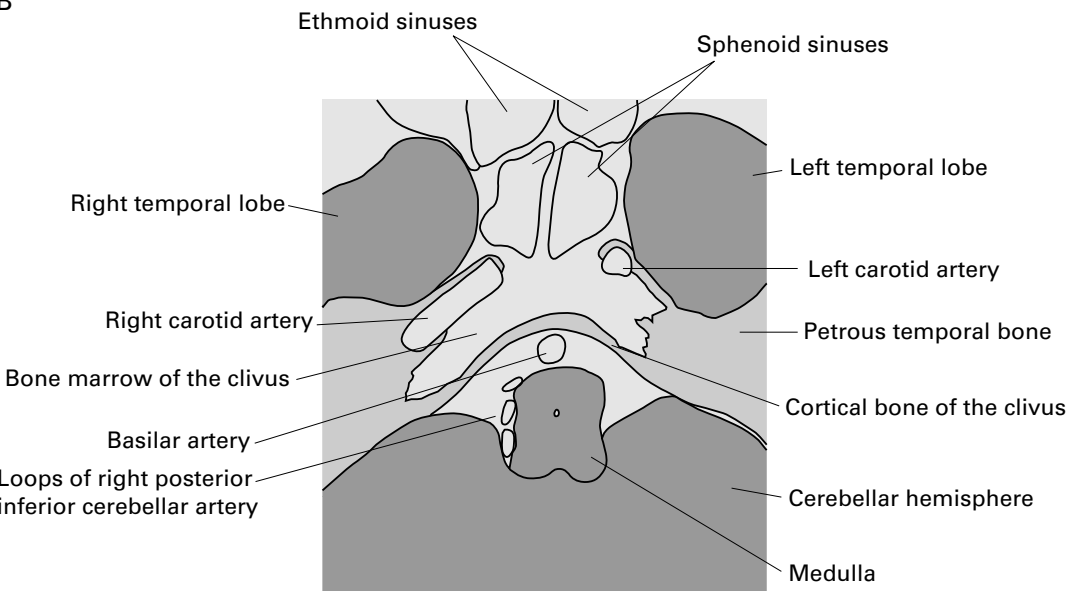

Figure 3 (A) Axial reformatted image through the posterior fossa just below the pontomedullary junction; shows the right posterior inferior cerebellar artery (PICA) compressing the medulla at the level of the right IX/Xth nerve complex. Because of their small size, the nerves are not visible on the scan, but were shown on contiguous axial images to be deviated around the right PICA. C, carotid arteries; B, basilar artery; black arrow, right PICA. (B) Line diagram showing MR features. 
A

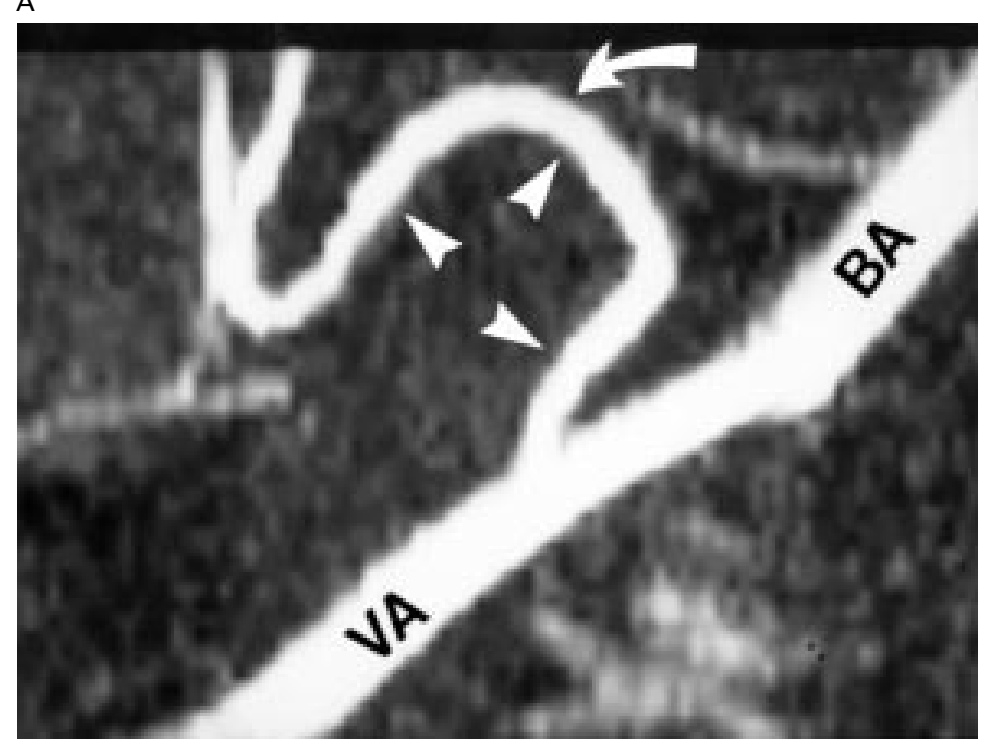

B

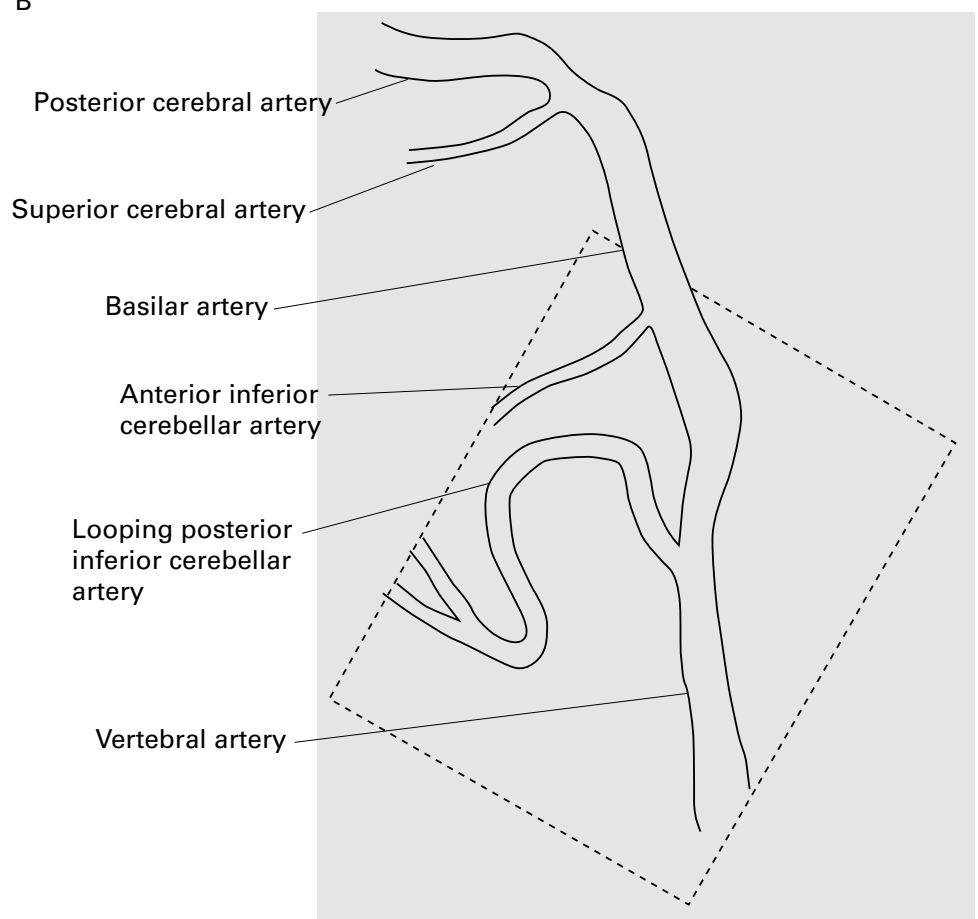

Figure 4 (A) Maximum intensity projection shows high looping PICA (arrowheads). The angiographic type image shows vascular anatomy only, but the site at which the artery compressed the nerve is indicated (curved arrow). VA, vertebral artery; $B A$, basilar artery. (B) Schematic diagram of right vertebrobasilar artery. Hatched box represents area of $M R$ image.

PROGRESS

Once the diagnosis of glossopharyngeal neuralgia had been made, she was commenced on carbamezapine. This produced little relief and unacceptable side effects occurred. Gabapentin was then instituted. This has resulted in dramatic relief of her symptoms. Microvascular compression is therefore not being considered at present but would be appropriate if her condition deteriorates.

\section{Discussion}

There has been debate as to whether vascular compression is a cause of "idiopathic" TN and GPN. With the advent of improved MR angio- graphic techniques and the publication of large neurosurgical series this has been widely, but not universally accepted. ${ }^{20}$ Further studies have implicated microvascular compression in the aetiology of hemifacial spasm, both in children and adults, ${ }^{21-23}$ essential hypertension, ${ }^{24}$ spasmodic torticollis, ${ }^{25} 26$ and episodic tinnitus. ${ }^{27}$

Although not acknowledged in most paediatric texts, there are a few published reports of idiopathic TN occurring in childhood. ${ }^{28}{ }^{29}$ Glossopharyngeal neuralgia has not been described in children.

The three children presented here all had severe pain, resulting in profound debilitation. Both patients with $\mathrm{TN}$ had been rendered effectively moribund by their symptoms. In each case delayed diagnosis and consequently delayed institution of appropriate treatment served to exacerbate the distress of both children and their families. Although the girl with GPN was less severely affected she had missed on average 12 days from school per term for years before the correct diagnosis was made. In her case, the diagnosis of recurrent otitis media was made with persistently normal examination findings on auroscopy.

Because of the poor acceptance of these disorders in the paediatric population, we explored many alternative diagnostic avenues and treatments before accepting $\mathrm{TN}$ as the diagnosis in Case 1, despite the fact that his pain, hyperaesthesia, and difficulty with facial hygiene were typical of the presentation of $\mathrm{TN}$ in later life. Our experience with Case 1 heightened awareness of neurovascular compression as a possible cause of symptoms in Case 2 . This, coupled with the availability of MR angiography, which showed vascular compression of the left Vth nerve, resulted in earlier neurosurgical referral.

There is no doubt that both patients experienced much more severe symptoms than most adult patients with TN. The severity of the pain probably relates to the fact that these cases represent the tip of the "symptomatic iceberg". Given the supposed rarity of the condition, many children and adolescents experiencing less severe pain may remain undiagnosed.

It is discouraging that although at each craniotomy there was evidence of microvascular compression, neither boy responded quickly to surgery and repeated procedures have been necessary to achieve symptom control. The literature suggests that $75-85 \%$ of adults respond to microvascular decompression, and relief is sustained for more than five years in two thirds of patients. ${ }^{13-15}$

There are a number of possible explanations for this. (1) Poor prognostic factors for a good surgical outcome consisting of severe symptoms, prolonged duration of pain, venous compression, and a younger age at presentation were all present in both patients. ${ }^{13} 1530$ (2) The neurovascular relations shown by MR angiography (MRA) may not correspond well to what is seen at craniotomy, as manipulation of the brain stem within the relatively "tight" posterior fossa of children may alter neurovascular relations, resulting in difficult identification of the critical compressing vessels even in expert 
hands. This may have been the situation with Case 2, where the vessel deforming the nerve on MRA was not appreciated as another vessel remote from the nerve on MRA, was found to be in close relation to the nerve at craniotomy. (3) The process of revascularisation, which results in recrudescence of symptoms owing to new vein formation, may be more aggressive in this age group. (4) It has been postulated that it is not the compression of the nerve per se but a resultant focus of demyelination that causes the typical neuralgic pain in TN. ${ }^{31} 32$ The regenerative process in the nerves of children, when myelination is not yet complete, may be different, accounting for their poor and somewhat delayed response to surgery.

\section{CONCLUSION}

Both glossopharyngeal and trigeminal neuralgia occur in children and can cause severe disability. These diagnoses should be considered in children presenting with paroxysmal facial, ear, or throat pain so that effective treatment can be given and investigation with MR angiography carried out. Children failing to respond to medical treatment should be considered for neurosurgery, if neurovascular compression is identified as the possible cause.

1 Zakrzewska JM. Trigeminal neuralgia. In: Zakrzewska JM, ed. Major problems in neurology. No. 28. London: Saunders, 1995.

2 International Association for the Study of Pain. Classification of chronic pain. Descriptions of chronic pain syndromes and definitions of pain terms. Pain 1986; 3(suppl):S49-57

3 Bruyn GW. Glossopharyngeal neuralgia. In: Clifford Rose $\mathrm{F}$, ed. Handbook of clinical neurology. Headache, Vol. 4(48) Amsterdam: Elsevier Science, 1986:459-73.

4 Fromm GH. Etiology and pathogenesis of trigeminal neuralgia. In: Fromm GH, ed. The medical and surgical management of trigeminal neuralgia. New York: Futura, 1987.

5 Kirsch E, Hausmann O, Kaim A, Gratzl O, Steinbrich W, Radu EW. Magnetic resonance imaging of vertebrobasilar ectasia in trigeminal neuralgia. Acta Neurochir 1996;138: 1295-8.

6 Burchiel KJ. Abnormal impulse generation in focally demyelinated trigeminal roots. $\mathcal{F}$ Neurosurg 1980;53:674-83.

7 Meaney JF, Watt JW, Eldridge PR, Whitehouse GH, Wells JC, Miles JB. Association between trigeminal neuralgia and mu, Miles JB. Association between trigeminal neuralgia and multiple sclerosis: role of magnetic resona
Neurol Neurosurg Psychiatry 1995;59:253-9.

8 Dandy WE. Surgery of the brain. In: Lewis D, ed. Practice of surgery. Hagerstown: WF Prior, 1945:177-200.

9 Jannetta PJ. Treatment of trigeminal neuralgia by suboccipital and transtentorial cranial operations. Clin Neurosurg 1977;24:538-49.

10 Kondo A. Follow-up results of using microvascular decompression for the treatment of glossopharyngeal neuralgia. $f$ Neurosurg 1998;88:221-5.
11 Olds MJ, Woods CI, Winfield JA. Microvascular decompression in glossopharyngeal neuralgia. Am f Otol 1995;16: 326-30.

12 Resnick DK, Jannetta PJ, Bissonnette D, Jho HD, Lanzino G. Microvascular decompression for glossopharyngeal neuralgia. Neurosurgery 1995;36:64-8.

13 Mendoza N, Illingworth RD. Trigeminal neuralgia treated by microvascular decompression: a long term follow-up study. Br f Neurosurg 1995;9:13-19.

14 Kondo A. Follow-up results of microvascular decompression in trigeminal neuralgia and hemifacial spasm. Neurosurgery 1997;40:46-51.

15 Barker FG II, Jannetta PJ, Bissonnette DJ, Larkins MV, Jho $\mathrm{HD}$. The long term outcome of microvascular decompression for trigeminal neuralgia. $N$ Engl f Med 1996;334: 1077-83.

16 Masur H, Papke K, Bongartz G, Vollbrecht K. The significance of three-dimensional MR-defined neurovascular compression for the pathogenesis of trigeminal neuralgia. $\mathcal{F}$ Neurol 1995;242:93-8.

17 Meaney JF, Eldridge PR, Dunn LT, Nixon TE, Whitehouse $\mathrm{GH}$, Miles JB. Demonstration of neurovascular compression in trigeminal neuralgia with magnetic resonance imaging. Comparison with surgical findings in 52 consecutive operative cases. F Neurosurg 1995;83:799-805.

18 Boecher-Schwarz HG, Bruehl K, Kessel G, Guenther M, Perneczky A, Stoeter P. Sensitivity and specificity of MRA in the diagnosis of neurovascular compression in patients with trigeminal neuralgia. A correlation of MRA and surgical findings. Neuroradiology 1998;40:88-95.

19 Weigel G. Kids with TN: it's rare but possible. TN Alert 1995-96; winter: 1,4

20 Adams CBT. Microvascular compression; an alternative view and hypothesis. $\mathcal{F}$ Neurosurg 1989;57:1-12.

21 Levy EI, Resnick DK, Jannetta PJ, Lovely T, Bissonnette DJ. Paediatric hemifacial spasm: the efficacy of microvascular decompression. Pediatr Neurosurg 1997;27:238-41.

22 Illingworth RD, Porter DG, Jakubowski J. Hemifacial spasm: a prospective long-term follow up of 83 cases treated by microvascular decompression at two neurosurgical centres in the United Kingdom. F Neurol Neurosurg Psychiatry 1996;60:72-7.

23 Kobata H, Kondo A, Kinuta Y, Iwasaki K, Nishioka T, Hasegawa K. Hemifacial spasm in childhood and adolescence. Neurosurgery 1995;26:710-14.

24 Geiger H, Naraghi R, Schobel HP, Frank H, Sterzel RB, Fahlbusch R. Decrease of blood pressure by ventrolateral medullary decompression in essential hypertension. Lancet 1998;352:446-9.

25 Jho HD, Jannetta PJ. Microvascular decompression for spasmodic torticollis. Acta Neurochir 1995;134:21-6.

26 Brunori A, Greco R, Bruni P, Delitala A, Chiappetta F. Surgical treatment of spasmodic torticollis: is there a role for microvascular decompression? With an illustrative case report. F Neurosurg Sci 1996;40:43-51.

27 Brookes GB. Vascular decompression surgery for severe tinnitus. Am f Otol 1996;17:569-76.

28 Mason WE, Kollros P, Jannetta PJ. Trigeminal neuralgia and its treatment in a 13 month old child: a review and case report. F Craniomandib Disord: Facial Oral Pain 1991;5: 213-16.

29 Haubner JM. An interesting case: trigeminal neuralgia. Northwest Dent 1992;71:35-6.

30 Bederson JB, Wilson CB. Evaluation of microvascular decompression and partial rhizotomy for treatment of trigeminal neuralgia. Neurosurgery 1989;65:28-31.

31 Calvin WH, Loeser JD, Howe JF. A neurophysiological theory for the pain mechanism of tic doloureux. Pain 1977; 3:147-54

32 Love S, Hilton DA, Coakham HB. Central demyelination of the Vth nerve root in trigeminal neuralgia associated with vascular compression. Brain Pathol 1998;8:1-11. 